\title{
Gene profiling of HepG2 cells following nitidine chloride treatment: An investigation with microarray and Connectivity Mapping
}

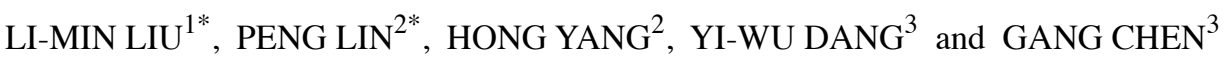 \\ ${ }^{1}$ Department of Toxicology, College of Pharmacy, Guangxi Medical University; \\ ${ }^{2}$ The Ultrasonics Division of Radiology Department and ${ }^{3}$ Department of Pathology, \\ The First Affiliated Hospital of Guangxi Medical University, Nanning, \\ Guangxi Zhuang Autonomous Region 530021, P.R. China
}

Received July 21, 2018; Accepted March 22, 2019

DOI: $10.3892 /$ or.2019.7091

\begin{abstract}
Nitidine chloride (NC), an inartificial bioactive alkaloid present in the root of Zanthoxylum nitidum (Roxb.) DC, is known for its versatile anti-inflammation and anticancer capabilities. The molecular mechanisms underlying its anticancer properties, however, remain obscure. The authors of the present study demonstrated the tumor suppressive effects of NC in a human liver cancer cell line using an MTT assay. The tumor suppressive capacity of NC was also analysed in a tumor xenograft nude mouse model. Changes in tumor cell gene expression profiles following $\mathrm{NC}$ treatment were detected by microarray; bioinformatics analysis demonstrated that differentially expressed genes were enriched in several cancer-associated pathways, including those initiated by transforming growth factor- $\beta$ and phosphatidylinositol 4,5-bisphosphate 3-kinase/RAC- $\alpha$ serine/threonine-protein kinase signaling. A Connectivity Map revealed that parthenolide, which has been identified previously as possessing anti-inflammatory and anticancer functions, was potentially extremely similar in molecular function to NC. By screening the data from The Cancer Genome Atlas project, eight genes that were upregulated in liver cancer and significantly suppressed by NC treatment were identified. Overexpression of these genes was closely associated with advanced tumor stage and poor differentiation
\end{abstract}

Correspondence to: Professor Yi-Wu Dang or Professor Gang Chen, Department of Pathology, The First Affiliated Hospital of Guangxi Medical University, 6 Shuangyong Road, Nanning, Guangxi Zhuang Autonomous Region 530021, P.R. China

E-mail: 87571061@qq.com

E-mail: chen_gang_triones@163.com

*Contributed equally

Key words: nitidine chloride, HepG2, proliferation, Connectivity Map, The Cancer Genome Atlas, bioinformatics analysis status. This combination of upregulated genes enabled successful identification and prediction of prognosis for liver cancer. The findings of the present study suggest that NC could inhibit the growth of liver cancer cells through several potential molecular targets and signaling pathways.

\section{Introduction}

Liver cancer is a serious global health problem, and it accounts for $4 \%$ of all new cancer cases and $6 \%$ of cancer-associated mortalities in men (1-3). Over 600,000 individuals succumb to liver cancer annually worldwide despite advances in treatment approaches like hepatic resection, image-guided tumor ablation and liver transplantation (4-6). The poor prognosis of liver cancer is due to its complex molecular mechanisms and limited molecular treatment targets (7). To date, few treatments with proven survival benefits exist for patients advanced liver cancer (8-10). The first drug approved for systemic therapy was a multiple kinase inhibitor termed sorafenib (11). Sorafenib has been plagued with toxic side-effects and limited efficacy $(12,13)$, therefore an urgent need for alternative liver cancer treatments persists (14-16). Doctors and medical scientists have gradually begun to recognize the anticancer therapeutic potential of natural compounds with their relatively few side-effects and curative effects for other diseases, including other types of cancer (17).

Nitidine chloride (NC), an ingredient well-known in traditional Chinese medicine, is present in the root of Zanthoxylum nitidum (Roxb.) DC (18). Previous studies revealed that NC could mediate a variety of physiological and pathological processes, including inflammation modulation (19), analgesia and tumor suppression (20,21). Previous studies on NC have focused on its antitumor effects in liver cancer. Liao et al (22) reported that $\mathrm{NC}$ inhibited the cell growth of liver cancer cells via the Janus-activated kinase 1/signal transducer and activator of transcription 3 (JAK1/STAT3) signaling pathway. NC also induced the apoptosis of liver cancer cells via pathways associated with p53, p21, Bax and Bcl-2 oncogenes (23). Research on the precise mechanisms by which $\mathrm{NC}$ functions remains in its early stages, with studies emphasizing the function of $\mathrm{NC}$ in 
tumor growth suppression (24-26). While NC is predicted to be of pivotal therapeutic significance, it may be challenging to uncover its mechanisms at the molecular level as well as from a systemic viewpoint.

To address these challenges, the cell growth inhibitory effect of $\mathrm{NC}$ on HepG2 cells was assessed in vitro; the tumor suppressive capacity of $\mathrm{NC}$ was also analysed in vivo in a murine model. A series of bioinformatic analyses were performed to uncover potential NC regulatory mechanisms based on the change in expression profiles following $\mathrm{NC}$ treatment on xenografted liver tumors. The clinical significance of several key genes associated with the function of $\mathrm{NC}$ in liver cancer were also analyzed. In doing so, the authors of the present study attempted to identify potential mechanisms of $\mathrm{NC}$ for the treatment of liver cancer.

\section{Materials and methods}

Preparation of NC and cell culture. NC with a purity $>95 \%$ was provided by Guangxi Traditional Chinese Medicine Institute (Nanning, China). NC was dissolved in dimethyl sulfoxide at a concentration of $10 \mathrm{mM}$ for subsequent testing. Human HepG2 cells were obtained from the Shanghai Cell Bank of the Chinese Academy of Sciences (Shanghai, China). HepG2 cells were cultured in Dulbecco's modified Eagle's medium (DMEM; Gibco; Thermo Fisher Scientific, Inc., Waltham, MA, USA) in a stable humidified atmosphere of 5\% $\mathrm{CO}_{2}$ at $37^{\circ} \mathrm{C}$. Authentication of the HepG2 cell line performed using short tandem repeat profiling.

Inhibitory effect of NC on HepG2 cells based on in vitro and in vivo models. The MTT method was performed to examine the inhibitory effect of NC on HepG2. Dimethyl sulfoxide was used to dissolve the purple formazan, the supernatant collected and then the absorbance of formazan was measured at a wavelength of $570 \mathrm{~nm}$. The inhibition rate was detected at 24,48 and $72 \mathrm{~h}$. A total of 32 six-week-old nude specific-pathogen-free BALB/c mice (16 males and 16 females; weighing 18-20 g) were purchased from the Shanghai Laboratory Animal Co., Ltd. (Shanghai, China) and housed in the Experimental Animal Center of Guangxi Medical University (Nanning, China). The mice were housed in specific-pathogen-free conditions in a controlled temperature of $24^{\circ} \mathrm{C}$, food and water were provided ad libitum. Mice were fed a sterile diet with a $12 / 12 \mathrm{~h}$ light/dark cycle. All procedures associated with the animal experiments were approved by the Animal Ethics Committee of Guangxi Medical University. The immunodeficient nude BALB/c mice were randomly assigned into four treatment groups, including a low-NC group $(2.5 \mathrm{mg} / \mathrm{kg} /$ day), a medium-NC group (5 mg/kg/day), a high-NC group $(10 \mathrm{mg} / \mathrm{kg} /$ day $)$ and a saline-only control group ( $0.2 \mathrm{ml}$ saline/day). Then, $0.2 \mathrm{ml} \mathrm{HepG2}$ cells was subcutaneously injected into the right axilla of these mice at a density of $1 \times 10^{10}$ cells $/ \mathrm{ml}$. A total of 7 days after the cells were injected, mice were intraperitoneally administered with $\mathrm{NC}(2.5,5$ or $10 \mathrm{mg} / \mathrm{kg} /$ day) or $0.2 \mathrm{ml}$ saline/day for 14 days. When the xenografted tumors reached $150-300 \mathrm{~mm}^{3}$, mice were transferred for further experimentation. On the day after tumor implantation, $\mathrm{NC}$ or saline was administered via intraperitoneal injection once per day for 14 days. The length and width of the xenografted tumors were measured at 7-day intervals using a caliper, and xenografted tumor size was calculated. Following the sacrifice of the animals, tumors were resected and weighed. Samples were fixed with $10 \%$ methanol at room temperature for $24 \mathrm{~h}$. Samples were cut into $0.5-\mu \mathrm{m}$-thick sections, prepared on $1 \mathrm{~mm}^{3}$ slides, and stained with hematoxylin and eosin at $37^{\circ} \mathrm{C}$ for $4 \mathrm{~min}$. The sections were then observed under a light microscope (magnification, x100 and x200) to examine morphology changes.

Gene profiling detected by microarray. The high-NC group and the saline group were used to microarray analysis. Human OneArray was provided by Aksomics, Inc. (Shanghai, China). The sample preparation, array hybridization and data analysis procedures were performed as previously reported $(27,28)$. Genes with a fold change (FC) of $\geq 1.5$ were considered differentially expressed genes (DEGs).

Gene enrichment analysis and network construction of DEGs following NC treatment. DEGs determined by the microarray analysis were further submitted for Gene Ontology (GO) and Kyoto Encyclopedia of Genes and Genomes (KEGG) analyses using the Database for Annotation, Visualization, and Integrated Discovery tool (version 6.8; https://david.ncifcrf. gov/). Functional enrichment terms at $\mathrm{P}<0.05$ were selected for further evaluation. To provide an intuitionistic and full-scale comprehension of the interaction network, the Search Tool for the Retrieval of Interacting Genes/Proteins database (https://string-db.org/; version 10.0) was utilized to construct a protein-protein interaction (PPI) network. Interactions among genes were calculated by setting a confidence threshold of $>0.9$.

Connectivity Map (cMap). To further elucidate potential regulatory mechanisms of the tumor suppressive function of $\mathrm{NC}$, a list of DEGs was submitted to the cMap database (https://portals. broadinstitute.org/cmap/; version, build 02) (29). Each imported query was compared with the predefined signatures of therapeutic compounds and ranked by the connectivity score (from -1 to 1), indicating the relative similarity of DEG profiles produced following NC treatment and the compound's treatment. A high positive connectivity score indicated compounds with functions similar to that of NC. Compounds with negative connectivity scores were dissimilar.

DEGs in liver cancer based on The Cancer Genome Atlas (TCGA). A list of DEGs in hepatocellular carcinoma, a type of liver cancer, and non-tumor samples was obtained from the online database, Gene Expression Profiling Interactive Analysis (GEPIA), to reveal potential mechanisms by which NC suppresses liver cancer (30). The set of DEGs met the following criteria: $\mid \log 2 \mathrm{FCl}>1$ and $\mathrm{q}<0.05$. Considering the significant anti-proliferation effects of $\mathrm{NC}$ in liver cancer cells, targets of $\mathrm{NC}$ are likely to be involved in the initiation and progression of liver cancer (23). Genes that were simultaneously upregulated in liver cancer and downregulated in the NC-treated groups were selected as key genes, as such genes that may be effective inhibitory targets of $\mathrm{NC}$ treatment for liver cancer. NC could exert its significant effects via these targets. Among the DEGs identified in the in-house microarray, the authors of the present study proposed that the key genes 
A

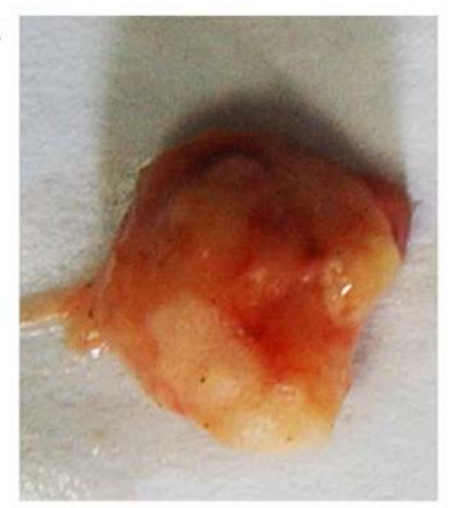

B

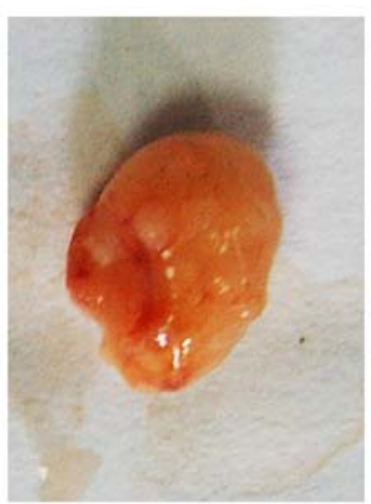

C

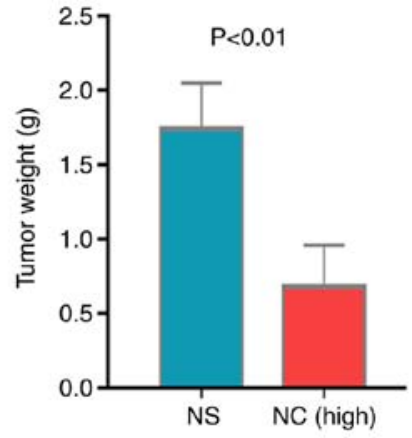

D

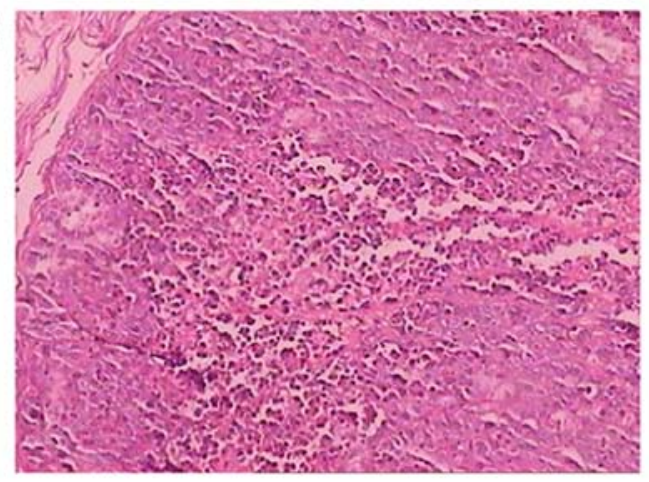

E

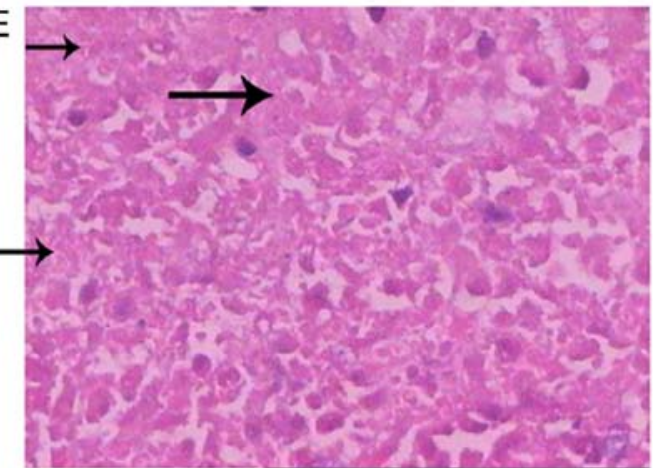

$\mathrm{F}$

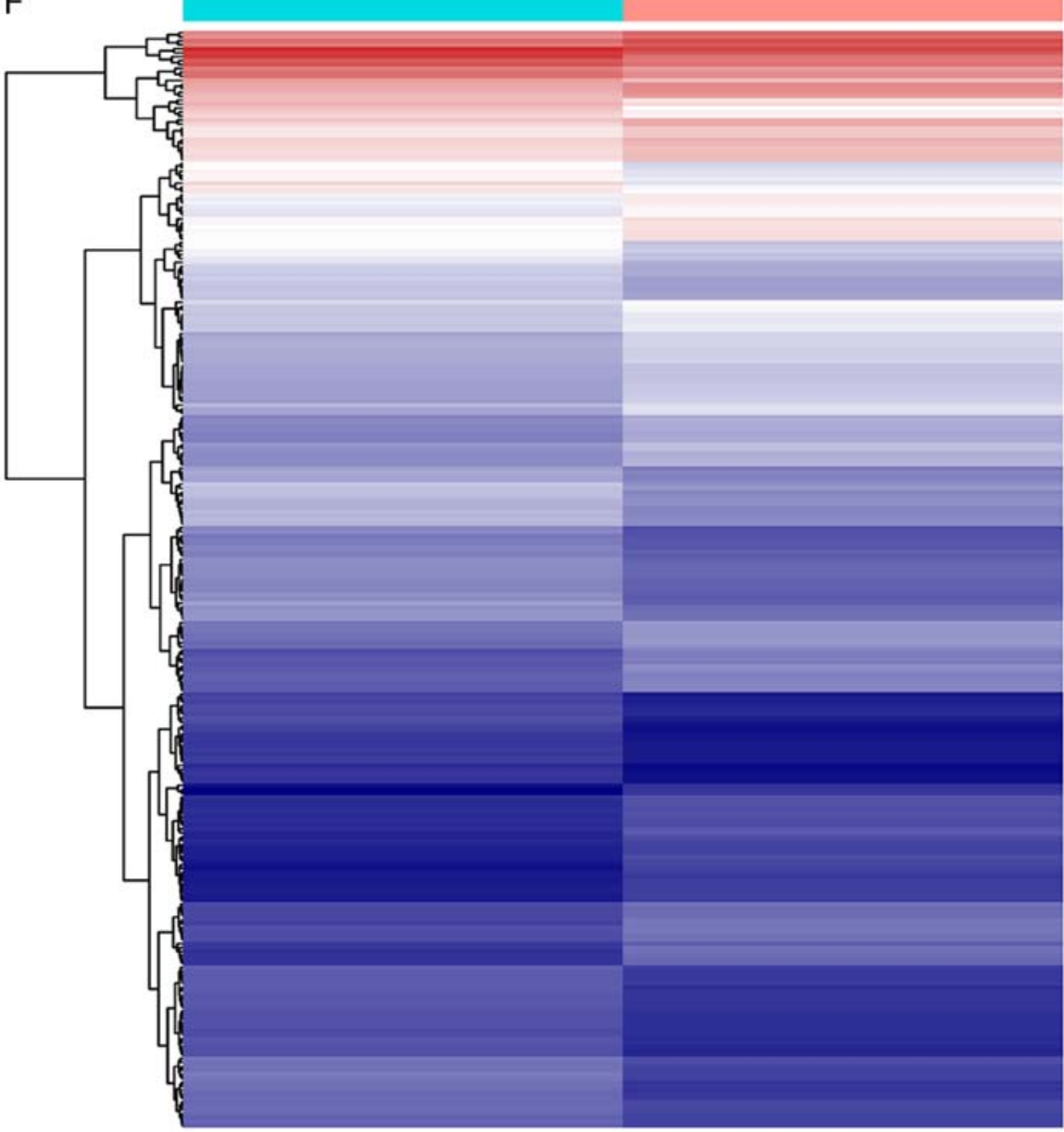

Group

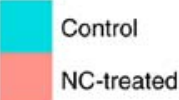

14

12

10

8

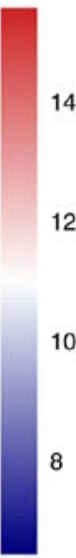

0

Figure 1. The tumor growth inhibitory effect of NC. Tumor excised from (A) untreated mice and (B) mice treated with NC. (C) Quantification of tumor weights from untreated mice and mice treated with NC. Hematoxylin and eosin staining images of (D) non-necrotic tumor cells in the control group and (E) necrotic tumor cells in the NC-treated group. The arrows indicate the stage of karyolysis where cells do not have nuclei. (F) A heat map of the differentially expressed genes between the control and NC-treated groups. The scale beside the heat map demonstrates that the color of each gene in the heat map corresponds with the expression level. NC, nitidine chloride. 
involved in liver cancer initiation and progression may be the effective targets of NC.

The Cancer Cell Line Encyclopedia (CCLE). The CCLE (version 2018; https://portals.broadinstitute.org/ccle), a database with detailed and comprehensive genetic characterizations, provides 28 publicly accessible liver cancer cell lines (namely SNU449, C3A, JHH7, HUH6, SNU761, LI7, HLF, JHH4, JHH1, JHH5, HUH7, SNU182, SNU398, SNU423, SNU387, SNU475, JHH6, SKHEP1, SNU886, SNU878, NCI-H684, PLC/PRF/5, Hep 3B2.1-7, HLE, PLCPRF5, HEPG2, HUH1 and JHH2 cell lines) with mRNA expression data. The expression levels of the aforementioned key genes were also validated using the data for liver cancer cell lines downloaded from the CCLE.

Development of diagnostic and prognostic signatures. Key genes were subjected to logistic regression analysis to develop a diagnostic index, which was more powerful than a single gene for distinguishing liver cancer from non-tumor samples. A prognostic risk score was constructed using the online database SurvExpress (version 2.0) (31) to validate the performance of multi-gene biomarkers for clinical outcomes in human cancers.

Determination of alteration status of key genes. To explore the underlying mechanisms relevant to genetic alterations in key genes, their alteration status was analyzed using the cBioPortal database (http://www.cbioportal.org/index.do) (32). The proportions of liver cancer patients with alterations to each gene and their survival status were obtained.

Statistical analysis. All statistical analyses were conducted using SPSS 24.0 (IBM Corp., Armonk, NY, USA). Associations between gene expression level and clinicopathological parameters of liver cancer patients were calculated using independent sample t-tests. One-way analysis of variance (ANOVA) followed by Bonferroni post hoc analysis was conducted to compare the difference of tumor weight when the control group was compared with low-NC, medium-NC and high-NC groups. The RNA-seq data obtained from TCGA and then $\log 2$-transformed for further analysis. A differentially expressed gene list was downloaded from GEPIA database. To explore the clinical significance of key genes, an independent t-test was used to compare the difference between HCC and non-tumor tissues. Receiver operating characteristic (ROC) curves and corresponding area under the ROC curves (AUC) were calculated for each gene to reveal the utility of genes for distinguishing liver cancer from non-tumor samples. Kaplan-Meier (K-M) plots of key genes were obtained from the GEPIA database. For all tests, $\mathrm{P}<0.05$ indicated that the difference between groups was statistically significant.

\section{Results}

Inhibitory effect of $\mathrm{NC}$ on HepG2 cells based on in vitro and in vivo models. The tumor growth inhibitory effect of NC indicated that the xenografts of untreated mice were significant heavier that of NC-treated mice (Fig. 1A and B). Tumor weight in the high-NC group was significantly lower compared with the saline group (Fig. 1C). Furthermore, there were many necrotic tumor cells in NC-treated group compared with control group (Fig. 1D and E). Microarray analysis revealed that 280 genes were altered post-NC treatment, including 138 upregulated and 142 downregulated genes (Fig. 1F). These genes were sent for further pathway and cMap inquiries. The cell growth inhibitory effect of $\mathrm{NC}$ on HepG2 cells in vitro was also confirmed. The higher NC concentrations markedly increased the inhibition rate of HepG2 cells (Fig. 2A). The body weight of nude mice was markedly changed following NC treatments (Fig. 2B). Tumor weights were significantly higher in the saline group compared with the medium-NC $(\mathrm{P}=0.012)$ and high-NC groups $(\mathrm{P}<0.001$; Fig. $2 \mathrm{C})$. The inhibition rate of $\mathrm{NC}$ increased in what appeared to be a dose dependent manner (Fig. 2D).

GO term enrichment and KEGG pathways. Results of gene functional enrichment analysis are summarized in Table I. Results of GO term enrichment analysis demonstrated that DEGs were most significantly associated with the biological process terms 'leukocyte migration', 'embryonic foregut morphogenesis' and 'response to antibiotic'. Unsurprisingly, an inflammation-associated term ranked first on the list. DEGs were also most significantly associated with the cellular component terms 'nucleus', 'nucleoplasm' and 'cytosol' (Fig. 3B). The top three most significant terms associated with molecular function were 'protein kinase binding', 'DNA binding' and 'protein binding'. The results of the KEGG enrichment analysis indicated that the transforming growth factor (TGF)- $\beta$ signaling pathway, the phosphatidylinositol 4,5-bisphosphate 3-kinase/RAC- $\alpha$ serine/threonine-protein kinase (PI3K-Akt) signaling pathway, and several tumor-associated signalling pathways were associated with the DEGs. These results depict the functional terms of DEGs. Using gene functional enrichment analysis, the potential molecular functions of NC were elucidated. NC may exert antitumor function via these pathways. 'TGF-beta signaling pathway', 'PI3K-Akt signaling pathway' and 'Arrhythmogenic right ventricular cardiomyopathy (ARVC)' were the three most significant KEGG pathways. The TGF- $\beta$ signaling pathway, which is involved in regulating biological processes, including cell growth, proliferation, differentiation, migration and apoptosis (33), was the top result of the enrichment analysis. Another important signaling pathway, PI3K-Akt, which has been demonstrated to significantly stimulate cell proliferation while inhibiting apoptosis (34), was suppressed by $\mathrm{NC}$ treatment.

Network analysis. The PPI network was constructed according to gene interactions with a confidence threshold $>0.9$. In total, 84 PPI associations in 61 nodes were identified. $R B X 1$ was the core gene in the network, suggesting it may be central to the action of NC on HepG2 cells (Fig. 3).

Analysis of NC by cMap. The results of the cMap analysis revealed a series of compounds with either similar or opposite effects to that of NC. The top ten results were parthenolide, trichostatin A, thapsigargin, disulfiram, vorinostat, ciclopirox, antimycin A, scriptaid, hycanthone and phenoxybenzamine, as presented in Table II. Among them, antimycin A may exhibit opposite effects to NC. The remaining compounds may have 

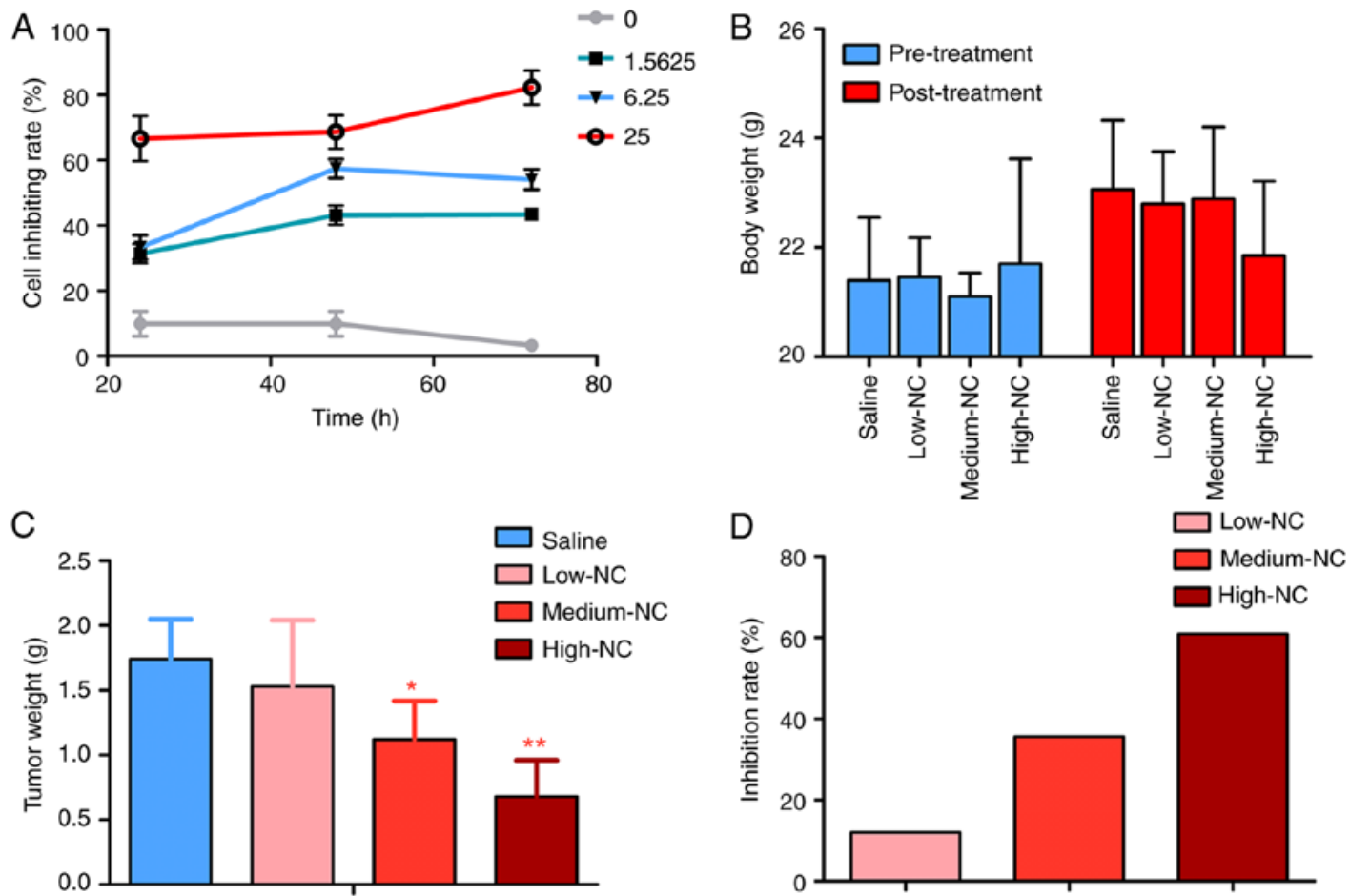

Figure 2. The tumor suppression ability of NC. (A) The proliferation ability was measured by MTT assays in HepG2 cells treated with NC. (B) Changes in body weight of nude mice prior to and following NC treatment. (C) Tumor weight and (D) inhibition rate of different groups. NC, nitidine chloride; low-NC, $2.5 \mathrm{mg} / \mathrm{kg} /$ day; medium-NC, $5 \mathrm{mg} / \mathrm{kg} / \mathrm{day}$; high-NC, $10 \mathrm{mg} / \mathrm{kg} / \mathrm{day} .{ }^{*} \mathrm{P}<0.05$ and ${ }^{* *} \mathrm{P}<0.01$, indicating significant difference.

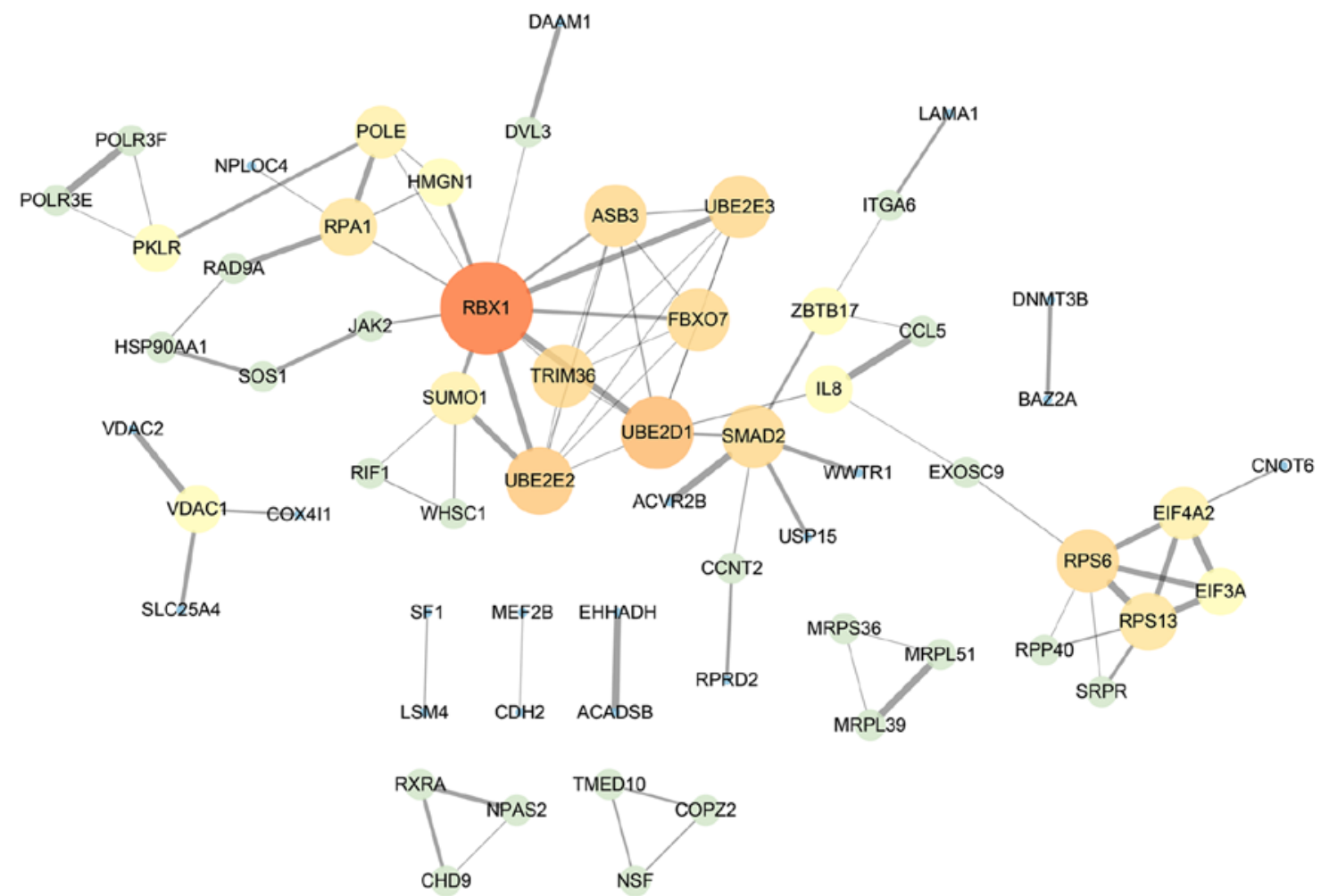

Figure 3. Protein association networks of differentially expressed genes. Interaction data obtained from the online database Search Tool for the Retrieval of Interacting Genes/Proteins. The size and brightness of the circle represent the degree of connection. The thickness of the lines represents the combined score.

similar functions to that of NC. These findings suggest that NC's efficacy may be based on activities similar or opposite to those of known compounds.
Key genes influenced by NC in liver cancer. By screening TCGA data from the GEPIA database, 2,218 DEGs were identified. Among them, 1,485 genes were upregulated and 
Table I. Gene functional enrichment analysis of differentially expressed genes.

\begin{tabular}{|c|c|c|c|c|}
\hline Category & Term & Count & Gene ratio $(\%)$ & P-value \\
\hline Biological process & GO:0050900-leukocyte migration & 7 & 2.702703 & 0.004638 \\
\hline Biological process & GO:0048617-embryonic foregut morphogenesis & 3 & 1.158301 & 0.005437 \\
\hline Biological process & GO:0046677-response to antibiotic & 4 & 1.544402 & 0.007613 \\
\hline Biological process & GO:0016337-single organismal cell-cell adhesion & 6 & 2.316602 & 0.009284 \\
\hline Biological process & GO:0006355-regulation of transcription, DNA-templated & 30 & 11.58301 & 0.015146 \\
\hline Biological process & GO:0060021-palate development & 5 & 1.930502 & 0.015870 \\
\hline Biological process & GO:1903959-regulation of anion transmembrane transport & 3 & 1.158301 & 0.019216 \\
\hline Biological process & GO:0031016-pancreas development & 3 & 1.158301 & 0.028706 \\
\hline Biological process & GO:0050821-protein stabilization & 6 & 2.316602 & 0.029689 \\
\hline Biological process & GO:0030335-positive regulation of cell migration & 7 & 2.702703 & 0.030093 \\
\hline Cellular component & GO:0005634-nucleus & 90 & 34.749030 & 0.001786 \\
\hline Cellular component & GO:0005654-nucleoplasm & 53 & 20.463320 & 0.001803 \\
\hline Cellular component & GO:0005829-cytosol & 56 & 21.621620 & 0.015614 \\
\hline Cellular component & GO:0005801-cis-Golgi network & 4 & 1.544402 & 0.015682 \\
\hline Cellular component & GO:0005737-cytoplasm & 81 & 31.27413 & 0.022047 \\
\hline Cellular component & GO:0030425-dendrite & 10 & 3.861004 & 0.026234 \\
\hline Cellular component & GO:0031012-extracellular matrix & 9 & 3.474903 & 0.034230 \\
\hline Cellular component & GO:0005913-cell-cell adherens junction & 9 & 3.474903 & 0.051794 \\
\hline Cellular component & GO:0005925-focal adhesion & 10 & 3.861004 & 0.059138 \\
\hline Cellular component & GO:0005667-transcription factor complex & 6 & 2.316602 & 0.096043 \\
\hline Molecular function & GO:0019901-protein kinase binding & 14 & 5.405405 & 0.001355 \\
\hline Molecular function & GO:0003677-DNA binding & 35 & 13.513510 & 0.006048 \\
\hline Molecular function & GO:0005515-protein binding & 134 & 51.737450 & 0.007031 \\
\hline Molecular function & GO:0042826-histone deacetylase binding & 6 & 2.316602 & 0.010778 \\
\hline Molecular function & GO:0044822-polyadenylated RNA binding & 24 & 9.266409 & 0.022243 \\
\hline Molecular function & GO:0046872-metal ion binding & 37 & 14.285710 & 0.042665 \\
\hline Molecular function & GO:0050660-flavin adenine dinucleotide binding & 4 & 1.544402 & 0.050953 \\
\hline Molecular function & GO:0061631-ubiquitin conjugating enzyme activity & 3 & 1.158301 & 0.054528 \\
\hline Molecular function & GO:0003682-chromatin binding & 10 & 3.861004 & 0.070868 \\
\hline Molecular function & GO:0042393-histone binding & 5 & 1.930502 & 0.075467 \\
\hline KEGG pathway & hsa04350-transforming growth factor- $\beta$ signaling pathway & 5 & 1.930502 & 0.030985 \\
\hline KEGG pathway & hsa04151-PI3K-Akt signaling pathway & 10 & 3.861004 & 0.055178 \\
\hline KEGG pathway & hsa05412-arrhythmogenic right ventricular cardiomyopathy & 4 & 1.544402 & 0.079302 \\
\hline KEGG pathway & hsa04022-cGMP-protein kinase G signaling pathway & 6 & 2.316602 & 0.086341 \\
\hline KEGG pathway & hsa04141-protein processing in endoplasmic reticulum & 6 & 2.316602 & 0.091529 \\
\hline KEGG pathway & hsa03013-RNA transport & 6 & 2.316602 & 0.096875 \\
\hline
\end{tabular}

PI3K-Akt, phosphatidylinositol 4,5-bisphosphate 3-kinase/RAC- $\alpha$ serine/threonine-protein kinase; KEGG, Kyoto Encyclopedia of Genes and Genomes; GO, Gene Ontology; hsa, Homo sapiens.

Table II. cMap analysis of the top 10 molecules similar to nitidine chloride.

\begin{tabular}{|c|c|c|c|c|c|c|}
\hline Rank & cMap name & Mean & $\mathrm{n}$ & Enrichment & P-value & Specificity \\
\hline 1 & Parthenolide & 0.684 & 4 & 0.944 & $<0.00001$ & 0.0359 \\
\hline 2 & Trichostatin A & 0.376 & 182 & 0.526 & $<0.00001$ & 0.2417 \\
\hline 3 & Thapsigargin & 0.632 & 3 & 0.940 & 0.00030 & 0.1019 \\
\hline 4 & Disulfiram & 0.556 & 5 & 0.817 & 0.00046 & 0.0116 \\
\hline 5 & Vorinostat & 0.319 & 12 & 0.536 & 0.00084 & 0.3970 \\
\hline 6 & Ciclopirox & 0.539 & 4 & 0.828 & 0.00133 & 0.0386 \\
\hline 7 & Antimycin A & -0.379 & 5 & -0.763 & 0.00142 & 0.0000 \\
\hline 8 & Scriptaid & 0.587 & 3 & 0.906 & 0.00174 & 0.0833 \\
\hline 9 & Hycanthone & 0.618 & 4 & 0.793 & 0.00362 & 0.0622 \\
\hline 10 & Phenoxybenzamine & 0.559 & 4 & 0.792 & 0.00364 & 0.2822 \\
\hline
\end{tabular}

cMap, Connectivity Map. 

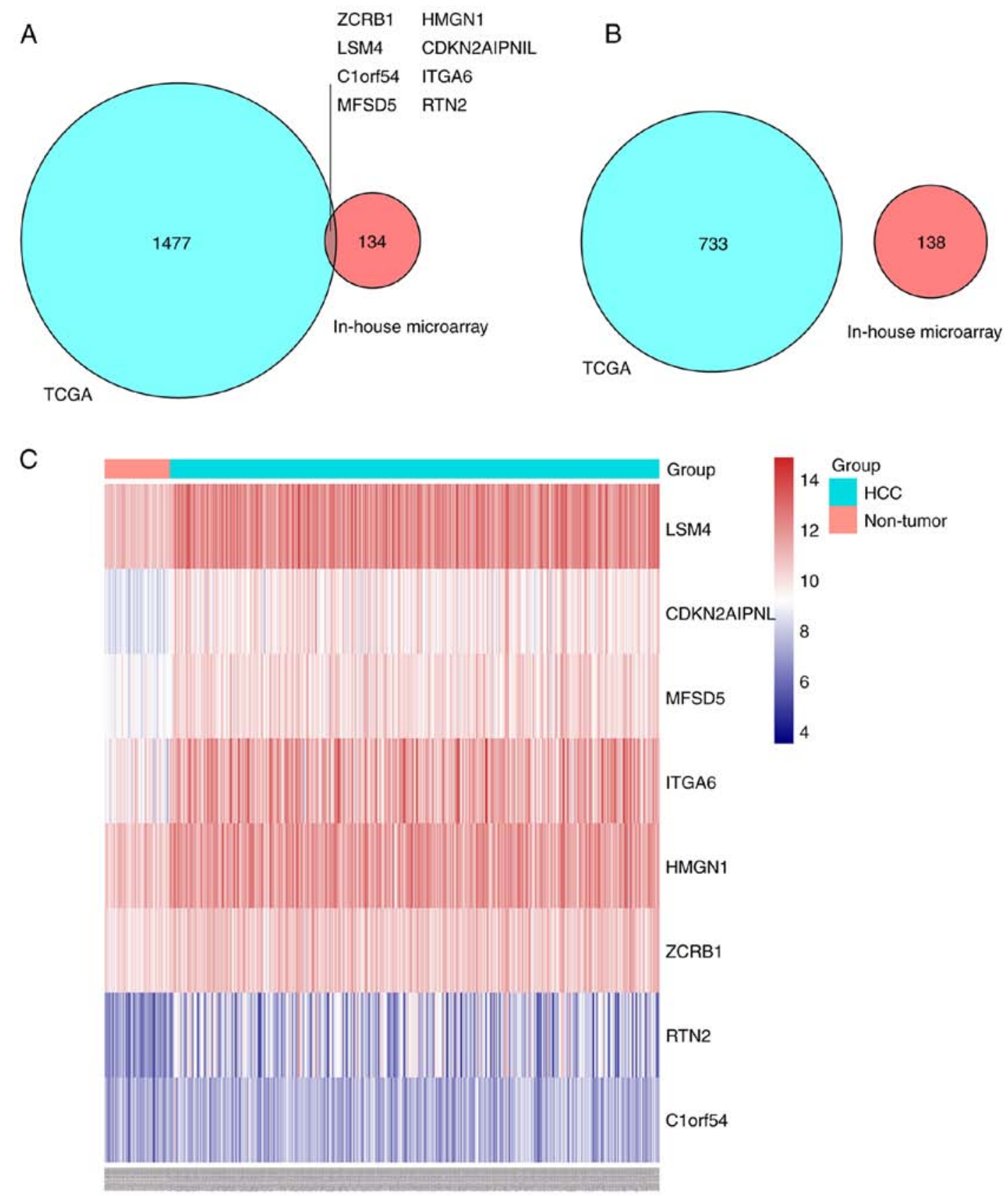

Figure 4. Intersection of DEGs of TCGA and DEGs of the in-house microarray. Venn diagrams of (A) genes that were differentially upregulated in liver cancer and inhibited in the NC-treated groups, and (B) genes that were differentially downregulated in liver cancer and overexpressed in the NC-treated groups. (C) A heat map presenting the expression profiles of the eight key genes. TCGA, The Cancer Genome Atlas; DEG, differentially expressed gene. LSM4, U6 snRNA-associated Sm-like protein LSm4; MFSD5, molybdate-anion transporter; HMGN1, non-histone chromosomal protein HMG-14; RTN2, reticulon-2; CDKN2AIPNL, CDKN2AIP N-terminal-like protein; ITGA6, integrin $\alpha$-6; ZCRB1, zinc finger CCHC-type and RNA-binding motif-containing protein 1; Clorf54, uncharacterized protein Clorf54.

733 downregulated. In the list of upregulated genes, it was determined that eight genes were significantly suppressed by NC treatment, including $L S M 4, C D K N 2 A I P N L, M F S D 5$, ITGA6, HMGN1, ZCRB1, RTN2 and ClORF54 (Fig. 4A). However, no genes that are differentially downregulated in liver cancer were overexpressed in the NC-treated groups (Fig. 4B). A heat map demonstrated the expression profiles of the eight genes; it revealed that the eight genes were upregulated in HCC tissues (Fig. 4C).

Clinical significance of the eight key genes. Revealing the clinical significance of these eight key genes could illuminate how NC suppresses tumors in HepG2 cells. The AUC values of these genes in the diagnosis of liver cancer, as well as the prediction of progress and prognosis of liver cancer were comprehensively analyzed. As presented in Fig. 5, these genes were overall significantly elevated in liver cancer compared with non-tumor tissues, although C1ORF54 expression levels only revealed a trend toward statistical significance. AUC values for the expression levels of the other seven genes were $>0.7$, illustrating a moderate potential for distinguishing liver cancer from non-tumor liver samples.

The patients with liver cancer were divided into different groups based on clinical parameters to analyze differences in 
A
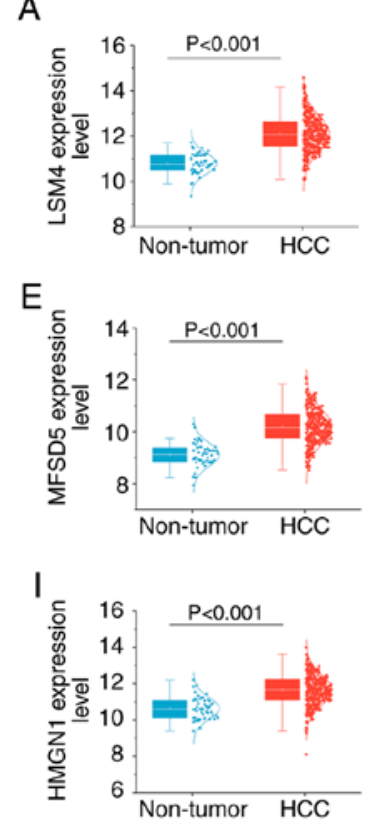

M

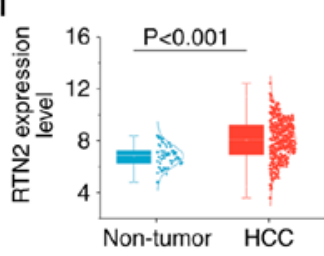

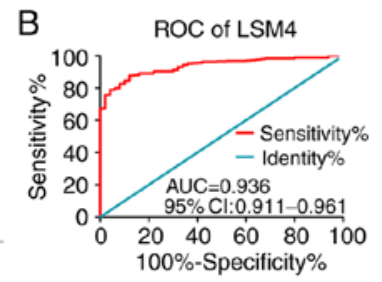
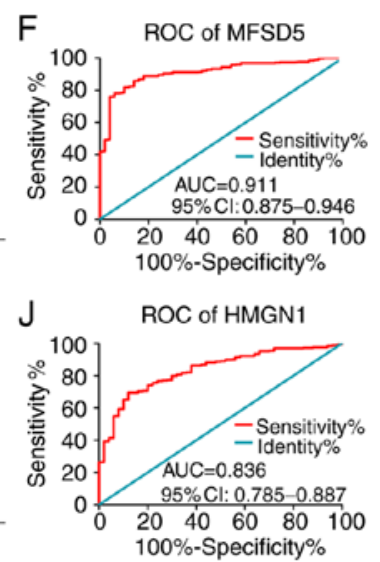

$\mathrm{N}$

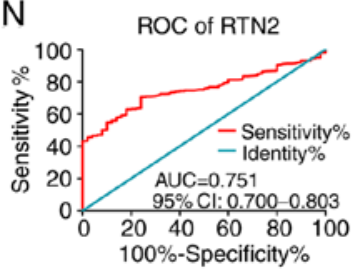

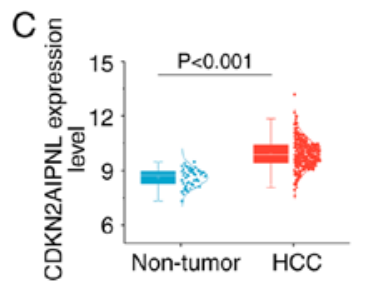

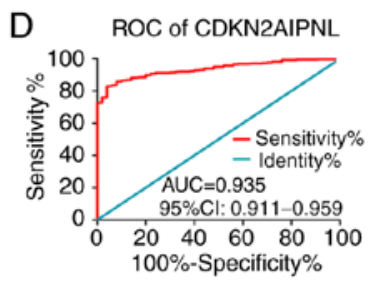

G
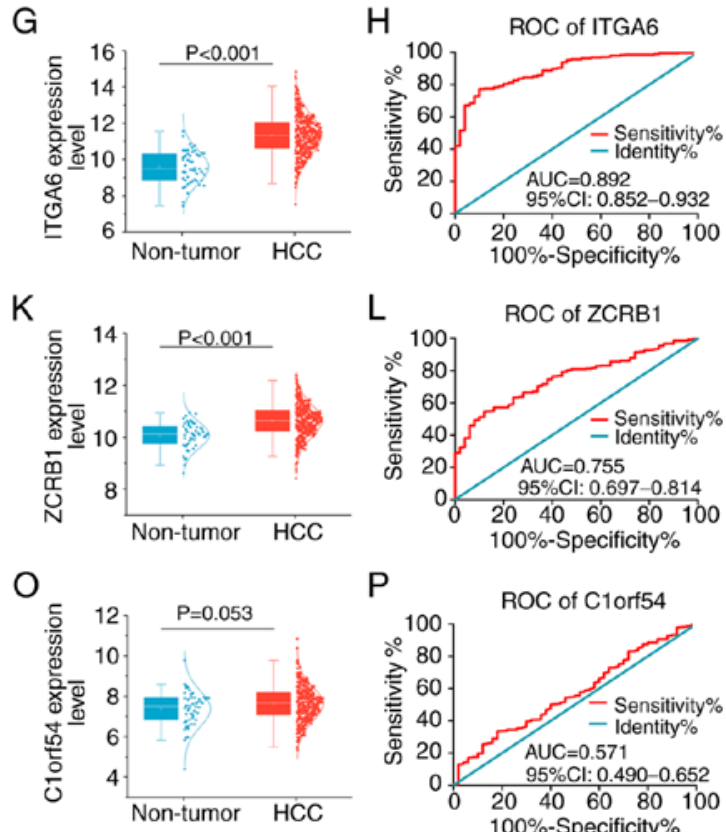

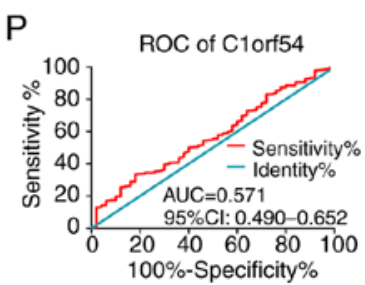

Figure 5. Expression levels and receiver operating characteristic curves of eight key genes between liver cancer and non-tumor samples. Gene expression was assessed in liver cancer and non-tumor samples. (A) Scatter plot and (B) ROC curve of LSM4 expression. (C) Scatter plot and (D) ROC curve of CDKN2AIPNL expression. (E) Scatter plot and (F) ROC curve of MFSD5 expression. (G) Scatter plot and (H) ROC curve of ITGA6 expression. (I) Scatter plot and (J) ROC curve of HMGN1 expression. (K) Scatter plot and (L) ROC curve of ZCRB1 expression. (M) Scatter plot and (N) ROC curve of RTN2 expression. (O) Scatter plot and (P) ROC curve of C1ORF54 expression. ROC, receiver operating characteristic; HCC, hepatocellular carcinoma; AUC, area under the ROC curves; CI, confidence interval; LSM4, U6 snRNA-associated Sm-like protein LSm4; MFSD5, molybdate-anion transporter; HMGN1, non-histone chromosomal protein HMG-14; RTN2, reticulon-2; CDKN2AIPNL, CDKN2AIP N-terminal-like protein; ITGA6, integrin $\alpha$-6; ZCRB1, zinc finger CCHC-type and RNA-binding motif-containing protein 1; C1orf54, uncharacterized protein C1orf54.

gene expression. Clinicopathological relevance analyses were performed for several variables, including age $(\geq 60$ years old vs. $<60$ years old; Fig. 6A), sex (male vs. female; Fig. 6B), pathologic stage (stage III/IV vs. stage I/II; Fig. 6C) and histologic grade (grade III/IV vs. grade I/II; Fig. 6D). HMGN1 was significantly overexpressed in younger patients. No noteworthy differences were observed between female and male patients with respect to the eight key genes. With respect to pathological stage, LSM4, MFSD5, ITGA6, HMGN1 and ZCRB1 were significantly upregulated in patients at advanced stages. As for histological grade, LSM4, CDKN2AIPNL, MFSD5 and $H M G N 1$ were significantly overexpressed in poorly differentiated tumor samples.

Survival curves were drawn using K-M analysis and a log-rank test was used to evaluate the prognostic values of these genes. Inferior overall survival was associated with high expression levels of $L S M 4, C D K N 2 A I P N L, M F S D 5$ or ZCRB; however, no difference in overall survival was observed when the expression levels of ITGA6, HMGN1, RTN2 and C1ORF54 were assessed (Fig. 6E-L). These findings indicated that these genes exerted an indispensable function in the initiation and progression of liver cancer. More importantly, these genes were significantly reduced in the NC-treated groups (Fig. 4A). These results suggest that $\mathrm{NC}$ may act as a tumor suppressor in liver cancer progression by inhibiting the activities of these genes.

Expression profiles in liver cancer cell lines. The authors of the present study analyzed gene expression values in the cell lines and found that all key genes had high expression levels with the exception of C1ORF54 (Fig. 7A).

Development of diagnostic index and prognostic index. A diagnostic index according to the following formula was constructed: Expression value of $L S M 4 \times 2.305+$ expression value of $C D K N 2 A I P N L \times 2.738+$ expression value of ITGA6x 2.336-expression value of ZCRB1x2.433-expression value of $C 1 O R F 54 \times 1.336$. The resulting diagnostic index significantly improved the ability of the authors to distinguish liver cancer from non-tumor tissues [AUC $=0.985$; 95\% confidence interval $(\mathrm{CI})=0.975-0.994 ; \mathrm{P}<0.001$; Fig. 7B]. These eight genes were also used to construct a prognostic signature using SurvExpress. The prognostic signature based on these genes was able to separate patients with liver cancer into two groups with significantly different prognostic statuses (hazard ratio=1.66; 95\% $\mathrm{CI}=1.16-2.36 ; \mathrm{P}=0.005$; Fig. $7 \mathrm{C}$ and $\mathrm{D})$. Patients in the high-risk group exhibited a worse prognosis. 
A
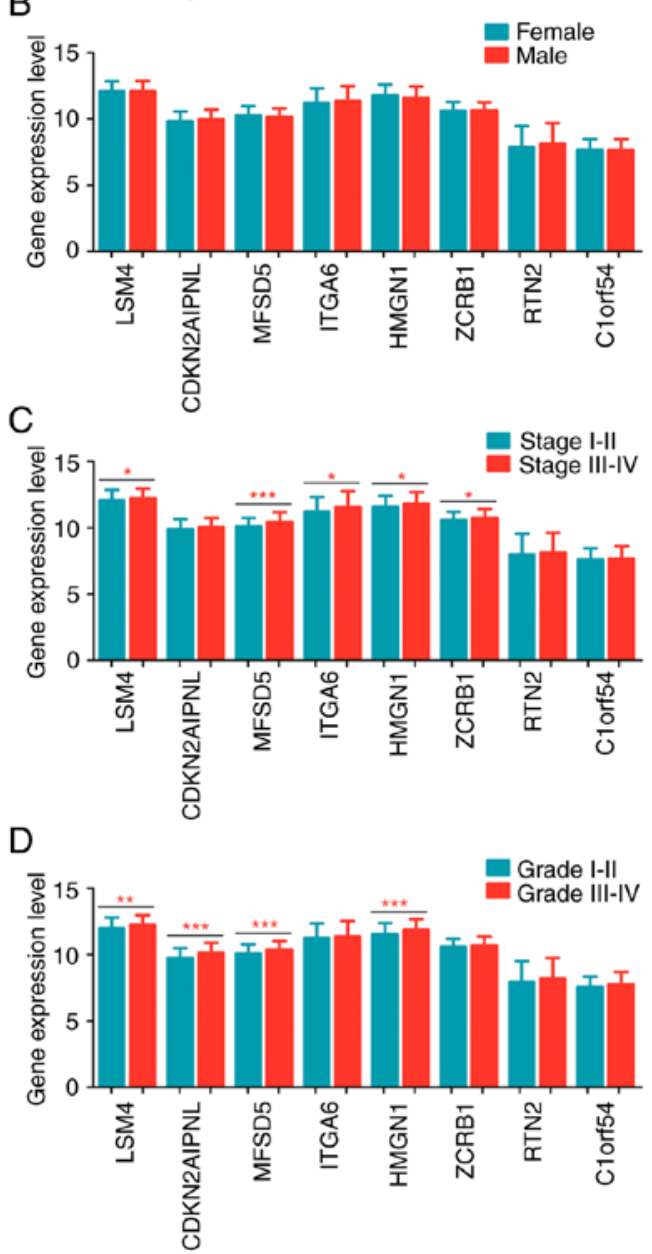
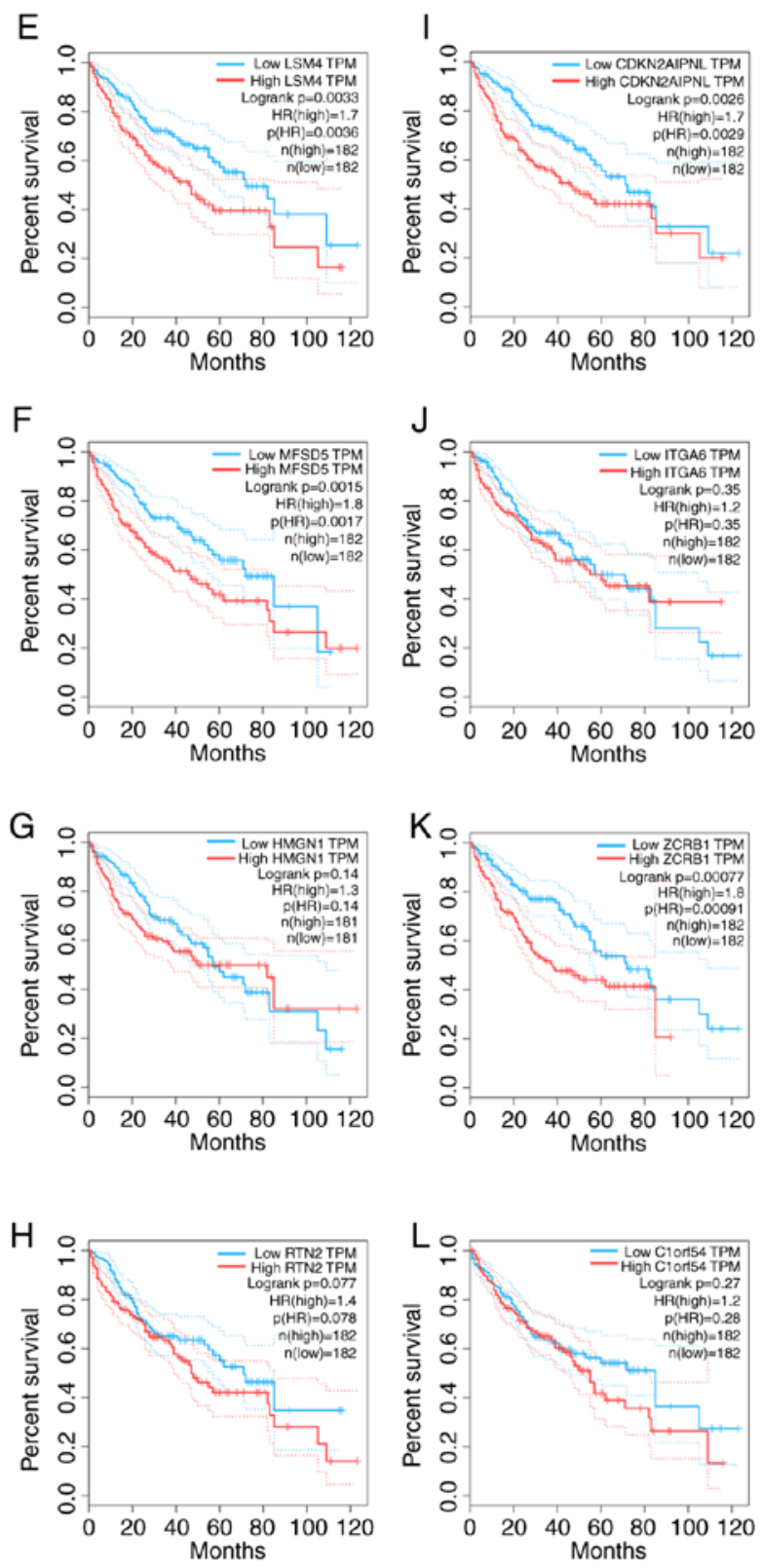

Figure 6. The clinical significance of eight key genes in liver cancer. Gene expression levels between (A) patients $<60$ and $\leq 60$ years old, (B) female and male, (C) tumor stages I-II and III-IV, and (D) tumor grades I-II and III-IV. Kaplan-Meier plots of (E) LSM4, (F) MFSD5, (G) HMGN1, (H) RTN2, (I) CDKN2AIPNL, (J) ITGA6, (K) ZCRB1 and (L) C1ORF54. HR, hazard ratio; LSM4, U6 snRNA-associated Sm-like protein LSm4; MFSD5, molybdate-anion transporter; HMGN1, non-histone chromosomal protein HMG-14; RTN2, reticulon-2; CDKN2AIPNL, CDKN2AIP N-terminal-like protein; ITGA6, integrin $\alpha$-6; ZCRB1, zinc finger CCHC-type and RNA-binding motif-containing protein 1; C1orf54, uncharacterized protein Clorf54; TPM, transcripts per million kilobases. ${ }^{*} \mathrm{P}<0.05,{ }^{* *} \mathrm{P}<0.01$ and ${ }^{* * *} \mathrm{P}<0.001$, indicating significant difference.

Genetic mutation status. Out of 440 patients with liver cancer, genetic alterations in the key genes was detected in $149(34 \%)$ of them using the online database cBioPortal (Fig. 8A). Among them, C1ORF54 was the gene with the highest alteration rate. However, K-M analysis yielded no differences between cases with and without alterations (Fig. 8B and 8C).

\section{Discussion}

Healthy cells carefully control cell proliferation, thereby ensuring homeostasis of the cell cycle, and uncontrolled proliferation is considered a fundamental trait of cancerous cells (35). It is crucial to understand the underlying mechanisms of proliferation and apoptosis to seek out and develop effective agents for combating the proliferation of cancer cells. In the present study, the authors identified DEGs between the high-NC and saline groups using microarray technology on HepG2 cell xenografts in nude mice. Next, functional annotation was conducted and functional connections between drugs to reveal the molecular characteristics of $\mathrm{NC}$ function were identified. TCGA database was mined and eight key genes with potential significant clinical value in modulating proliferation were uncovered. These eight genes may be the effectors upon which NC applies its antitumor function. These findings could 

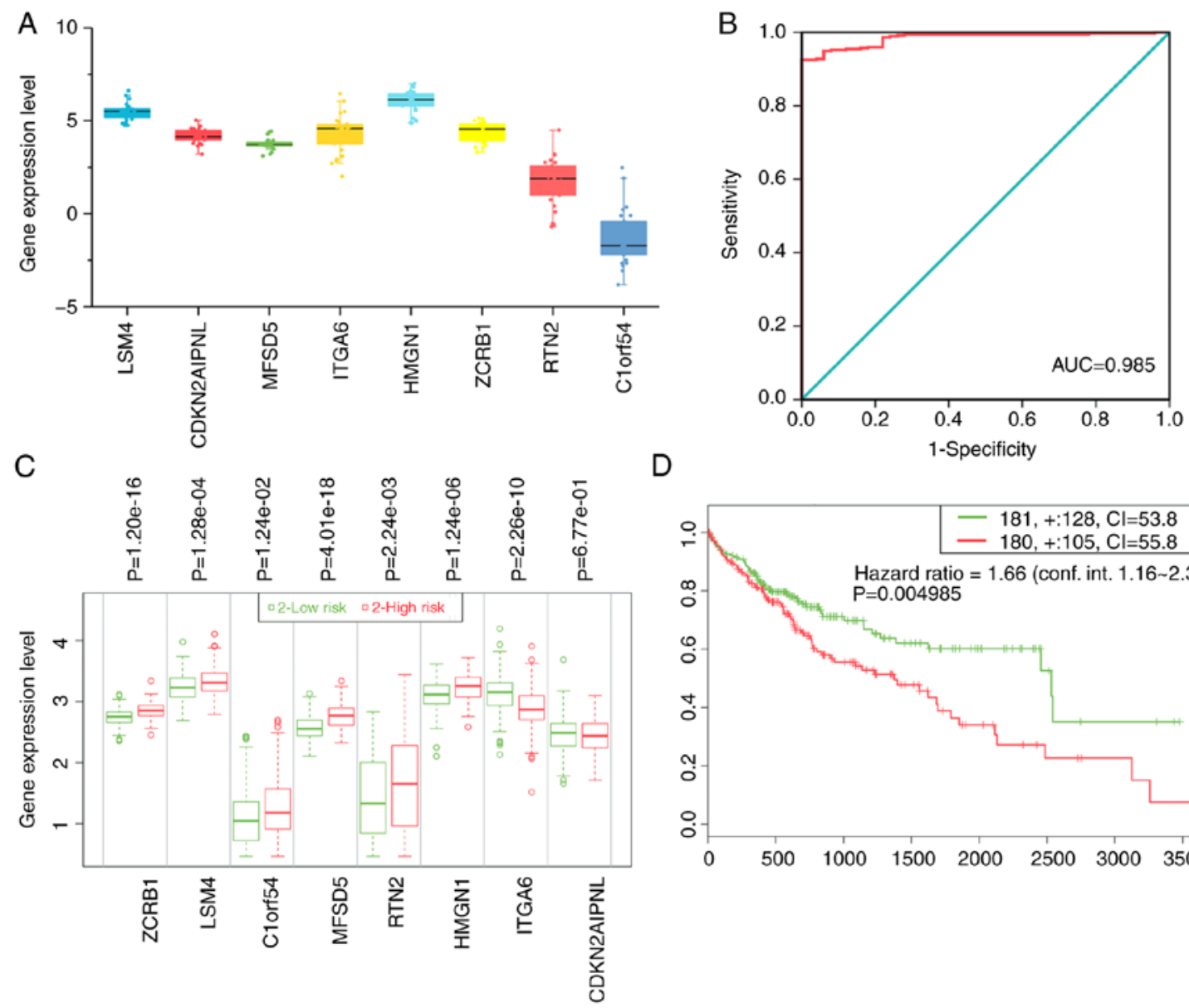

D

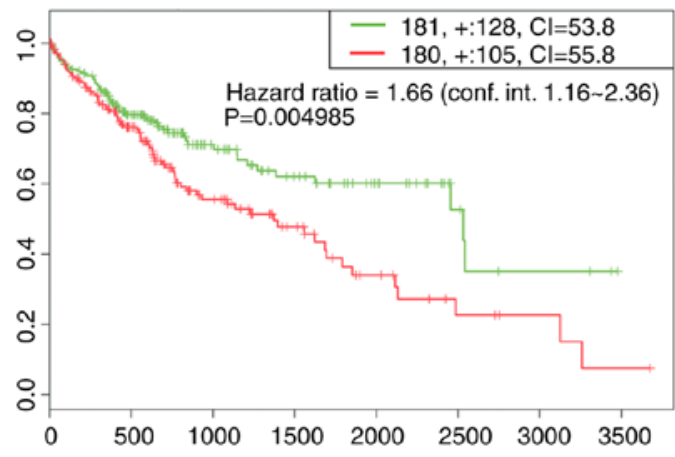

Figure 7. The overall clinical value of key genes. (A) Relative expression levels of key genes in liver cancer cells. (B) ROC of diagnostic index. (C) The expression levels of the eight key genes and (D) the Kaplan-Meier plot in the high- and low-risk groups. ROC, receiver operating characteristic; AUC, area under the ROC curves; CI, confidence interval; LSM4, U6 snRNA-associated Sm-like protein LSm4; MFSD5, molybdate-anion transporter; HMGN1, non-histone chromosomal protein HMG-14; RTN2, reticulon-2; CDKN2AIPNL, CDKN2AIP N-terminal-like protein; ITGA6, integrin $\alpha$-6; ZCRB1, zinc finger CCHC-type and RNA-binding motif-containing protein 1; Clorf54, uncharacterized protein Clorf54.

help identify novel potential targets for the treatment of liver cancer by NC.

Recent studies have demonstrated that $\mathrm{NC}$ is an important antitumor compound $(24,36)$. Of all the functions in which NC has been implicated, the authors of the present study consider inhibiting proliferation and inducing apoptosis are the most important. The process of liver cancer treatment by NC may involve multiple genes. Thus, DEGs (identified by analyzing gene expression prior to and after NC treatment) were studied to provide a theoretical basis for delineating the mechanism by which NC inhibits proliferation and tumor cell cycles, and induces apoptosis. GO and KEGG pathway analyses predicted that the downregulated and upregulated genes were associated with inflammation- or cancer-associated terms. The first biological process term, 'leukocyte migration,' indicated that the immunologic microenvironment was significantly influenced by NC. Indeed, NC is a well-known inhibitor of inflammation $(18,19)$.

The PI3K-Akt signaling pathway, which regulates cell growth, survival, metabolism and apoptosis, was also implicated $(37,38)$. A growing body of research has indicated that inhibiting PI3K/Akt signaling could contribute to the suppression of liver cancer development (39-41). Of interest, inflammation- and tumor-progression-associated pathways were prominent in the results. The leading enriched KEGG pathway, TGF- $\beta$, has also been considered to act as a protumorigenic factor in liver cancer by remodeling chronic inflammation-mediated hepatocarcinogenesis, and promoting liver cancer growth, metastasis and angiogenesis (42-44). The findings of the present study suggest that NC acts against liver cancer molecular targets and the tumor growth environment.

In addition to interfering with signaling pathways, NC may also have molecular functions similar to those of established compounds. Based on the results of validation experiments using cMap, NC was considered to exert a similar function to parthenolide, which could explain the molecular function of NC. Parthenolide has been revealed to possess anti-inflammatory and anticancer activities (45-47). Carlisi et al (48) reported that parthenolide sensitizes liver cancer cells to tumor necrosis factor-associated, apoptosis-inducing ligands through the inhibition of STAT3 activation. Of note, NC also inhibited the activation of the JAK1/STAT3 signaling pathway in liver cancer (22). This comparison further supports an anti-inflammatory and antitumor capacity of NC on liver cancer cells. The results of the present study also provide clues to other biological $\mathrm{NC}$ mechanisms against liver cancer. 
A

Altered in 149 (34\%) of 440 sequenced cases/patients ( 440 total)

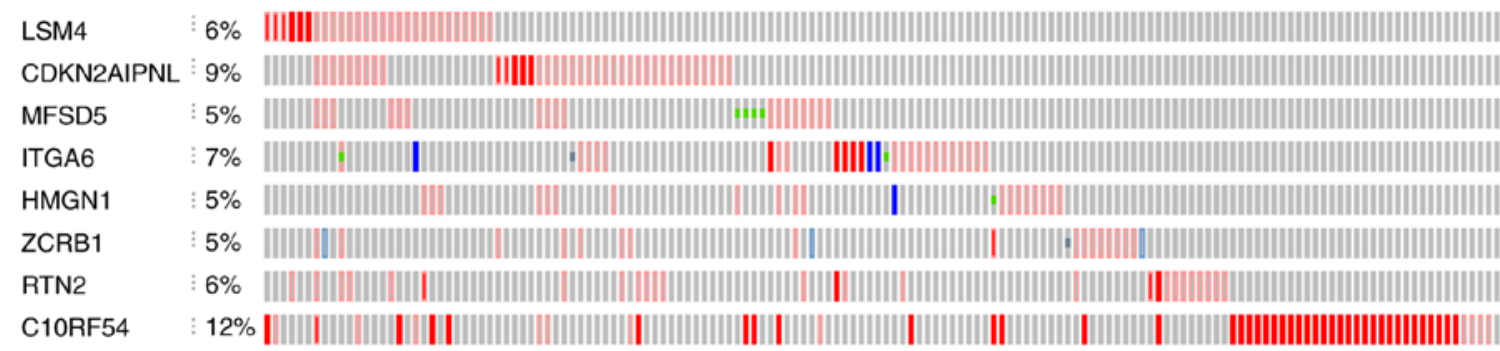

Genetic alteration | No alterations \Amplification \Deep deletion \mRNA upregulation [ mRNA downregulation

- Truncating mutation (putative passenger) = Missense mutation (putative passenger)

B

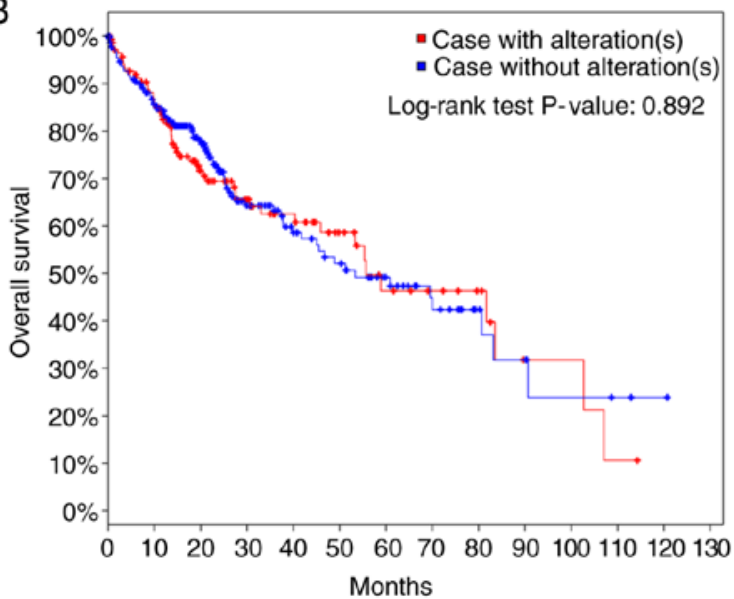

C

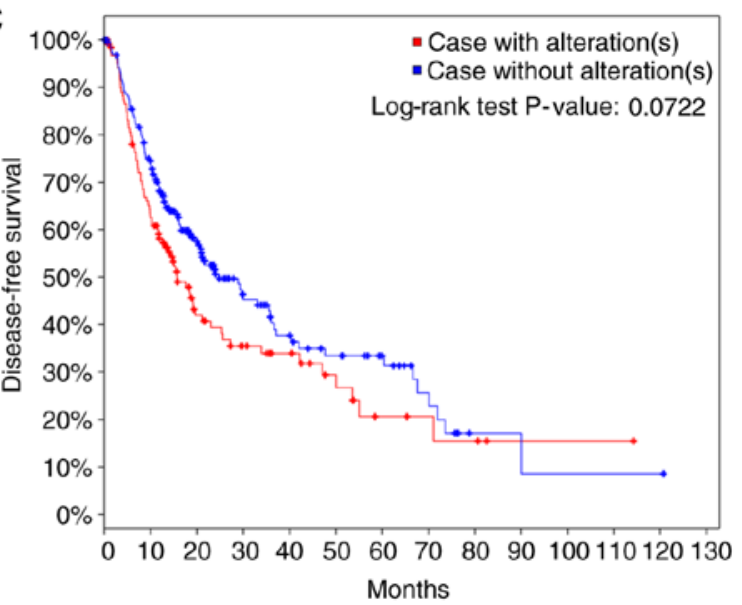

Figure 8. Alteration status of eight key genes. (A) Overview of the alterations status of key genes. The overall percentage (34\%) represents patients with one or more genetic mutation, while individual percentages represent patients with a mutation in one gene. (B) Overall and (C) disease-free survival of cases with alterations and cases without alterations. LSM4, U6 snRNA-associated Sm-like protein LSm4; MFSD5, molybdate-anion transporter; HMGN1, non-histone chromosomal protein HMG-14; RTN2, reticulon-2; CDKN2AIPNL, CDKN2AIP N-terminal-like protein; ITGA6, integrin $\alpha-6$; ZCRB1, zinc finger CCHC-type and RNA-binding motif-containing protein 1; C1orf54, uncharacterized protein C1orf54.

Among the DEGs identified in the in-house microarray, key liver cancer initiation and progression genes suspected of being targeted by $\mathrm{NC}$ were processed. $\mathrm{NC}$ was observed to suppress a total of eight overexpressed genes in liver cancer. Consistent with the hypothesis of the present study, high expression levels of certain genes were significantly associated with advanced stage and grade of liver cancer, and could serve as potential biomarkers for predicting the overall survival of patients with liver cancer. NC may enact its anti-proliferative function through these genes. A study by our group also provided certain clues as to NC functional mechanisms (49). Ou et al (23) demonstrated that $\mathrm{NC}$ induced apoptosis in ovarian cancer cells by activating the Fas signaling pathway. Kim et al (46) reported that NC suppressed the growth of acute myeloid leukemia cells by inhibiting the phosphorylation of AKT and ERK. A study has also proposed that the upregulation of $p 53, p 21$ and $B a x$, and the downregulation of $\mathrm{Bcl}$-2 mediate $\mathrm{NC}$ apoptotic function in liver cancer (23). Many studies have addressed different targets for NC antitumor function $(25,26,50-52)$. The present study integrated the classical functional experiments and big data mining, which could provide novel insights into the molecular characteristics of NC. Generally, cancer can be regarded as a disorder in the equilibrium between cell growth and death, and these genes are frequently involved in cancer disorders $(49,3)$. In recent years, the research and development of new drugs have been based on the pharmacological action of drugs on multiple targets and through multiple pathways to achieve long-term low toxicity and to reduce drug resistance $(53,54)$. Clinical significance was also explored by treating the eight key genes as a set. Additional survival analysis revealed that the gene set was highly correlated with a reduced overall survival. This result provides a useful conceptual framework for understanding the complex biology of NC effects on liver cancer.

However, there are several limitations of the present study. First, only two cell plates used for the microarray. Hence, the preliminary study is required a confirmation in the future. Second, several bioinformatics analyses were performed based on prediction algorithms, future in vitro or in vivo experiments are needed to explore the exact regulatory mechanisms.

In conclusion, the present study determined that NC exhibited tumor growth inhibitory effects in vitro and in vivo. It was also demonstrated that different key genes are potentially involved in NC-mediated antitumor function, implicating several prospective proliferative signaling pathways. The present study demonstrates the antitumor function of $\mathrm{NC}$ and provides insights into the potential complex molecular functions of $\mathrm{NC}$ against liver cancer. In brief, $\mathrm{NC}$ has the potential 
for future clinical application when its in-depth molecular mechanisms have been fully explored.

\section{Acknowledgments}

Not applicable.

\section{Funding}

The present study was financially supported by funds from the National Natural Science Foundation of China (grant no.NSFC81560489), the Natural Science Foundation of Guangxi, China (grant no. 2017GXNSFAA198017), the Innovation Project of Guangxi Graduate Education (grant no. YCSW2018104), the Guangxi First-class Discipline Project for Pharmaceutical Sciences (grant no. GXFCDP-PS-2018), the Guangxi Medical University Training Program for Distinguished Young Scholars, and the Medical Excellence Award Funded by the Creative Research Development Grant from the First Affiliated Hospital of Guangxi Medical University.

\section{Availability of data and materials}

The datasets used during the present study are available from the corresponding author upon reasonable request.

\section{Authors' contributions}

YWD and GC conceived and designed the experiments. PL and LML performed the experiments. PL and HY analyzed the data. PL, HY, LML and GC wrote the manuscript. All authors read and approved the final manuscript.

\section{Ethics approval and consent to participate}

All procedures associated with the animal experiments were approved by the Animal Ethics Committee of Guangxi Medical University (Nanning, China).

\section{Patient consent for publication}

Not applicable.

\section{Competing interests}

The authors declare no competing interests.

\section{References}

1. Siegel RL, Miller KD and Jemal A: Cancer statistics, 2018. CA Cancer J Clin 68: 7-30, 2018.

2. Laursen L: A preventable cancer. Nature 516: S2-3, 2014.

3. Fujiwara N, Friedman SL, Goossens N and Hoshida Y: Risk factors and prevention of hepatocellular carcinoma in the era of precision medicine. J Hepatol 68: 526-549, 2018.

4. Torre LA, Bray F, Siegel RL, Ferlay J, Lortet-Tieulent J and Jemal A: Global cancer statistics, 2012. CA Cancer J Clin 65 87-108, 2015

5. El-Serag HB: Epidemiology of viral hepatitis and hepatocellular carcinoma. Gastroenterology 142: 1264-1273 el, 2012.

6. Liu Z, Wang C, Jiao X, Zhao S, Liu X, Wang Y and Zhang J: miR-221 promotes growth and invasion of hepatocellular carcinoma cells by constitutive activation of NFkB. Am J Transl Res 8: 4764-4777, 2016.
7. Wong $\mathrm{CH}$, Wong $\mathrm{CS}$ and Chan SL: Targeting angiogenic genes as a therapeutic approach for hepatocellular carcinoma. Curr Gene Ther 15: 97-108, 2015.

8. Forner A, Reig M and Bruix J: Hepatocellular carcinoma. Lancet 391: 1301-1314, 2018.

9. Wu CH, Lan CH, Wu KL, Wu YM, Jane WN, Hsiao M and Wu HC: Hepatocellular carcinoma-targeted nanoparticles for cancer therapy. Int J Oncol 52: 389-401, 2018.

10. Shi JY, Ma LJ, Zhang JW, Duan M, Ding ZB, Yang LX, Cao Y, Zhou J, Fan J, Zhang X, et al: FOXP3 is a HCC suppressor gene and Acts through regulating the TGF- $\beta / \mathrm{Smad} 2 / 3$ signaling pathway. BMC Cancer 17: 648, 2017.

11. Raoul JL, Kudo M, Finn RS, Edeline J, Reig M and Galle PR: Systemic therapy for intermediate and advanced hepatocellular carcinoma: Sorafenib and beyond. Cancer Treat Rev 68: 16-24, 2018.

12. Nada Y, Rashad N, Eissa M, Ghonaim A, Farag K, Saadawi I, Sheha A, El Gewaity M and Abdel-Rahman O: Outcomes of treatment with sorafenib in Egyptian patients with hepatocellular carcinoma: A retrospective cohort study. Expert Rev Gastroenterol Hepatol 12: 99-107, 2018.

13. Bruix J, Takayama T, Mazzaferro V, Chau GY, Yang J, Kudo M, Cai J, Poon RT, Han KH, Tak WY, et al: Adjuvant sorafenib for hepatocellular carcinoma after resection or ablation (STORM): A phase 3, randomised, double-blind, placebo-controlled trial. Lancet Oncol 16: 1344-1354, 2015.

14. Kudo M: Systemic therapy for hepatocellular carcinoma: 2017 update. Oncology 93 (Suppl 1): S135-S146, 2017.

15. Yuan W, Sun Y, Liu L, Zhou B, Wang S and Gu D: Circulating lncRNAs serve as diagnostic markers for hepatocellular carcinoma. Cell Physiol Biochem 44: 125-132, 2017.

16. Wu J, Li A, Yang J, Lu Y and Li J: Efficacy and safety of TACE in combination with sorafenib for the treatment of TACE-refractory advanced hepatocellular carcinoma in Chinese patients: A retrospective study. Onco Targets Ther 10: 2761-2768, 2017.

17. Mondal S, Bandyopadhyay S, Ghosh MK, Mukhopadhyay S, Roy S and Mandal C: Natural products: Promising resources for cancer drug discovery. Anticancer Agents Med Chem 12: 49-75, 2012.

18. Hu J, Zhang WD, Liu RH, Zhang C, Shen YH, Li HL, Liang MJ and Xu XK: Benzophenanthridine alkaloids from Zanthoxylum nitidum (Roxb.) DC, and their analgesic and anti-inflammatory activities. Chem Biodivers 3: 990-995, 2006.

19. Khan H, Hadda TB and Touzani R: Diverse therapeutic potential of nitidine, a comprehensive review. Curr Drug Metab 19: 986-991, 2018.

20. Fang Z, Tang Y, Jiao W, Xing Z, Guo Z, Wang W, Shi B, Xu Z and Liu Z: Nitidine chloride inhibits renal cancer cell metastasis via suppressing AKT signaling pathway. Food Chem Toxicol 60: 246-251, 2013.

21. Kim LH, Khadka S, Shin JA, Jung JY, Ryu MH, Yu HJ, Lee HN, Jang B, Yang IH, Won DH, et al: Nitidine chloride acts as an apoptosis inducer in human oral cancer cells and a nude mouse xenograft model via inhibition of STAT3. Oncotarget 8: 91306-91315, 2017.

22. Liao J, Xu T, Zheng JX, Lin JM, Cai QY, Yu DB and Peng J: Nitidine chloride inhibits hepatocellular carcinoma cell growth in vivo through the suppression of the JAK1/STAT3 signaling pathway. Int J Mol Med 32: 79-84, 2013.

23. Ou X, Lu Y, Liao L, Li D, Liu L, Liu H and Xu H: Nitidine chloride induces apoptosis in human hepatocellular carcinoma cells through a pathway involving p53, p21, Bax and Bcl-2. Oncol Rep 33: 1264-1274, 2015.

24. Chen S, Yang L and Feng J: Nitidine chloride inhibits proliferation and induces apoptosis in ovarian cancer cells by activating the Fas signalling pathway. J Pharm Pharmacol 70: 778-786, 2018.

25. Cheng Z, Guo Y, Yang Y, Kan J, Dai S, Helian M, Li B, Xu J and Liu C: Nitidine chloride suppresses epithelial-to-mesenchymal transition in osteosarcoma cell migration and invasion through Akt/GSK-3//Snail signaling pathway. Oncol Rep 36: 1023-1029, 2016.

26. Lin J, Shen A, Chen H, Liao J, Xu T, Liu L, Lin J and Peng J: Nitidine chloride inhibits hepatic cancer growth via modulation of multiple signaling pathways. BMC Cancer 14: 729, 2014.

27. Zhang Y, Dang YW, Wang X, Yang X, Zhang R, Lv ZL and Chen G: Comprehensive analysis of long non-coding RNA PVT1 gene interaction regulatory network in hepatocellular carcinoma using gene microarray and bioinformatics. Am J Transl Res 9: 3904-3917, 2017. 
28. Zhang Y, He RQ, Dang YW, Zhang XL, Wang X, Huang SN, Huang WT, Jiang MT, Gan XN, Xie Y, et al: Comprehensive analysis of the long noncoding RNA HOXA11-AS gene interaction regulatory network in NSCLC cells. Cancer Cell Int 16 : 89, 2016.

29. Lamb J, Crawford ED, Peck D, Modell JW, Blat IC, Wrobel MJ, Lerner J, Brunet JP, Subramanian A, Ross KN, et al: The Connectivity Map: Using gene-expression signatures to connect small molecules, genes, and disease. Science 313: 1929-1935, 2006.

30. Tang Z, Li C, Kang B, Gao G, Li C and Zhang Z: GEPIA: A web server for cancer and normal gene expression profiling and interactive analyses. Nucleic Acids Res, 2017.

31. Aguirre-Gamboa R, Gomez-Rueda H, Martinez-Ledesma E, Martínez-Torteya A, Chacolla-Huaringa R, RodriguezBarrientos A, Tamez-Peña JG and Treviño V: SurvExpress: An online biomarker validation tool and database for cancer gene expression data using survival analysis. PLoS One 8: e74250, 2013.

32. Gao J, Aksoy BA, Dogrusoz U, Dresdner G, Gross B, Sumer SO, Sun Y, Jacobsen A, Sinha R, Larsson E, et al: Integrative analysis of complex cancer genomics and clinical profiles using the cBioPortal. Sci Signal 6: pl1, 2013.

33. Rao $S$ and Mishra L: Targeting TGF- $\beta$ signaling in liver cancer. Hepatology: Dec 14, 2018 (Epub ahead of print). doi: 10.1002/hep.30426.

34. Liu M, Wang J, Qi Q, Huang B, Chen A, Li X and Wang J: Nitidine chloride inhibits the malignant behavior of human glioblastoma cells by targeting the PI3K/AKT/mTOR signaling pathway. Oncol Rep 36: 2160-2168, 2016

35. Hanahan D and Weinberg RA: Hallmarks of cancer: The next generation. Cell 144: 646-674, 2011

36. Kim LH, Khadka S, Shin JA, Jung JY, Ryu MH, Yu HJ, Lee HN, Jang B, Yang IH, Won DH, et al: Nitidine chloride acts as an apoptosis inducer in human oral cancer cells and a nude mouse xenograft model via inhibition of STAT3. Oncotarget 8 : 91306-91315, 2017.

37. Wang Z, Jiang W, Zhang Z, Qian M and Du B: Nitidine chloride inhibits LPS-induced inflammatory cytokines production via MAPK and NF-kappaB pathway in RAW 264.7 cells. J Ethnopharmacol 144: 145-150, 2012.

38. Lamarca A, Mendiola M and Barriuso J: Hepatocellular carcinoma: Exploring the impact of ethnicity on molecular biology. Crit Rev Oncol Hematol 105: 65-72, 2016.

39. Bupathi M, Kaseb A, Meric-Bernstam F and Naing A: Hepatocellular carcinoma: Where there is unmet need. Mol Oncol 9: 1501-1509, 2015.

40. Zhu YJ, Zheng B, Wang HY and Chen L: New knowledge of the mechanisms of sorafenib resistance in liver cancer. Acta Pharmacol Sin 38: 614-622, 2017.

41. Liu L, Liao JZ, He XX and Li PY: The role of autophagy in hepatocellular carcinoma: Friend or foe. Oncotarget 8: 57707-57722, 2017.

42. Mazzocca A, Antonaci S and Giannelli G: The TGF- $\beta$ signaling pathway as a pharmacological target in a hepatocellular carcinoma. Curr Pharm Des 18: 4148-4154, 2012.
43. Neuzillet C, de Gramont A, Tijeras-Raballand A, de Mestier L, Cros J, Faivre S and Raymond E: Perspectives of TGF- $\beta$ inhibition in pancreatic and hepatocellular carcinomas. Oncotarget 5: 78-94, 2014.

44. Yang P, Li QJ, Feng Y, Zhang Y, Markowitz GJ, Ning S, Deng Y, Zhao J, Jiang S, Yuan Y, et al: TGF- $\beta$-miR-34a-CCL22 signaling-induced Treg cell recruitment promotes venous metastases of HBV-positive hepatocellular carcinoma. Cancer Cell 22: 291-303, 2012.

45. Li-Weber M, Palfi K, Giaisi M and Krammer PH: Dual role of the anti-inflammatory sesquiterpene lactone: Regulation of life and death by parthenolide. Cell Death Differ 12: 408-409, 2005.

46. Kim SL, Park YR, Lee ST and Kim SW: Parthenolide suppresses hypoxia-inducible factor-1alpha signaling and hypoxia induced epithelial-mesenchymal transition in colorectal cancer. Int J Oncol 51: 1809-1820, 2017.

47. Zhang X, Chen Q, Liu J, Fan C, Wei Q, Chen Z and Mao X: Parthenolide Promotes Differentiation of Osteoblasts Through the Wnt/ $\beta$-catenin signaling pathway in inflammatory environments. J Interferon Cytokine Res 37: 406-414, 2017.

48. Carlisi D, D'Anneo A, Angileri L, Lauricella M, Emanuele S, Santulli A, Vento R and Tesoriere G: Parthenolide sensitizes hepatocellular carcinoma cells to TRAIL by inducing the expression of death receptors through inhibition of STAT3 activation. J Cell Physiol 226: 1632-1641, 2011.

49. Liu LM, Xiong DD, Lin P, Yang H, Dang YW and Chen G: DNA topoisomerase 1 and $2 \mathrm{~A}$ function as oncogenes in liver cancer and may be direct targets of nitidine chloride. Int J Oncol 53: 1897-1912, 2018

50. Li P, Yan S, Dong X, Li Z, Qiu Y, Ji C, Zhang J, Ji M, Li W, Wang $\mathrm{H}$, et al: Cell cycle arrest and apoptosis induction activity of nitidine chloride on acute myeloid leukemia cells. Med Chem 14: 60-66, 2018

51. Liu N, Li P, Zang S, Liu Q, Ma D, Sun X and Ji C: Novel agent nitidine chloride induces erythroid differentiation and apoptosis in CML cells through c-Myc-miRNAs axis. PLoS One 10: e0116880, 2015.

52. Zhai H, Hu S, Liu T, Wang F, Wang X, Wu G, Zhang Y, Sui M, Liu $\mathrm{H}$ and Jiang L: Nitidine chloride inhibits proliferation and induces apoptosis in colorectal cancer cells by suppressing the ERK signaling pathway. Mol Med Rep 13: 2536-2542, 2016.

53. Mody K and Abou-Alfa GK: Systemic therapy for advanced hepatocellular carcinoma in an evolving landscape. Curr Treat Options Oncol 20: 3, 2019.

54. Abou-Alfa GK, Meyer T, Cheng AL, El-Khoueiry AB, Rimassa L, Ryoo BY, Cicin I, Merle P, Chen Y, Park JW, et al: Cabozantinib in patients with advanced and progressing hepatocellular carcinoma. N Engl J Med 379: 54-63, 2018.

This work is licensed under a Creative Commons Attribution-NonCommercial-NoDerivatives 4.0 International (CC BY-NC-ND 4.0) License. 